\title{
GLAST and Gamma-Ray Bursts
}

\author{
Robert D. Preece \\ University of Alabama in Huntsville, Department of Physics \\ 320 Sparkman Drive, Huntsville, AL 35805, USA
}

\begin{abstract}
The two instruments aboard the Gamma-Ray Large Area Space Telescope (GLAST) mission will make unprecedented spectral observations of gamma-ray bursts. I will discuss what new science we can expect from GLAST.
\end{abstract}

\section{Introduction}

The GLAST Observatory will fill the gap left by the demise of the instruments on board the Compton Gamma-Ray Observatory (CGRO) in May 2000, especially the Energetic Gamma-Ray Experiment Telescope (EGRET), and extend upon their successes. Although there have been several new hard X-Ray and lowenergy gamma-ray experiments flown or about to fly recently, the Large Area Telescope (LAT) on GLAST is suited to take EGRET's place observing the gamma-ray sky at energies above $20 \mathrm{MeV}$ with very good statistics. Indeed, the LAT's proposed capabilities are superior to those of EGRET and represent more than an order of magnitude better look at the high-energy universe. At the same time, a GLAST gamma-ray burst monitor (GBM) will be included on the observatory to provide a way to put new GRB observations by the LAT in context with the known statistics and characteristics of bursts as seen by, for example, BATSE. I will review the general capabilities of these two instruments and then discuss how they will together advance the current science of GRBs, primarily using spectroscopy.

\subsection{The LAT on GLAST}

To greatly improve upon EGRET, the LAT will have a much larger effective area as well as much reduced deadtime. For detection efficiency, the LAT consists of modular silicon-strip detectors, backed by CsI calorimeters. Pair tracking will be done electronically with little of the temporal overhead that contributed to EGRET's deadtime and none of the consumables. The GLAST LAT has a field of view about twice as wide (more than 2.5 steradians), and sensitivity about 50 times that of EGRET at $100 \mathrm{MeV}$ and even more at higher energies. Its two year limit for source detection in an all-sky survey is $1.6 \times 10^{-9}$ photons $\mathrm{cm}^{-2} \mathrm{~s}^{-1}$ (at energies $>100 \mathrm{MeV}$ ). It will be able to locate sources to positional accuracies of 30 arc seconds to 5 arc minutes.

The LAT baseline design for the GLAST tracker consists of a four-by-four array of tower modules. Each tower module consists of interleaved planes of silicon-strip detectors (SSDs) and tracker lead converter sheets. SSDs are able 
to more precisely track the electron or positron produced from the initial gammaray than previous types of detectors. SSDs will have the ability to determine the location of an object in the sky to within 0.5 to 5 arc minutes. The pair conversion signature is also used to help reject the much larger background of charged cosmic rays. The high intrinsic efficiency and reliability of this technology enables straight forward event reconstruction and excellent resolution with small tails. These ease-of-use properties will maximize the mission science return for guest observers.

In each module, there are 19 pairs of planes of silicon - in each pair, one plane has the strips oriented in the "x-direction", while the other has the strips oriented in the perpendicular "y-direction". When a particle interacts in the silicon, its position on the plane can therefore be determined in two dimensions. The third dimension of the track is determined by analyzing signals from adjacent planes, as the particle travels down through the telescope towards the calorimeter.

The calorimeter design for GLAST consists of cesium-iodide CsI(Tl) bars, arranged in a segmented manner, giving both longitudinal and transverse information about the energy deposition pattern. Once a gamma ray penetrates through the anti-coincidence shield, the silicon-strip tracker and lead converter planes, it then passes into the CsI calorimeters. The CsI blocks are arranged in two perpendicular directions, to provide additional positional information about the shower. The anti-coincidence detector consists of segmented plastic scintillator tiles, read out by wave-shifting fibers and photo-multiplier tubes. The segmentation is designed to avoid the self-veto problem of EGRET at high energies while still providing high cosmic-ray rejection.

\subsection{The GBM on GLAST}

The GLAST Burst Monitor should be looked upon in the light of GLAST's primary goal, which is spectroscopy. The instrument consists of two types of detectors, $12 \mathrm{NaI}$ and two BGO. The NaI detectors will have some localization ability to enable the Observatory to determine whether a given transient source is outside the broad field of view of the LAT. Depending upon the relative interest in the event, whether it is particularly hard for example, the LAT can decide on board whether the spacecraft should repoint. In addition, the LAT can respond to a rapid ( $5 \mathrm{~ms}$ ) trigger signal that the GBM will provide. Primarily, the GBM provides the spectroscopy context by which the expected new results from the LAT will be compared.

The requirement and goal of the lower energy threshold of the GBM NaI detectors are 10 and $5 \mathrm{keV}$, respectively. By design, the high-energy threshold will be modest at $1 \mathrm{MeV}$ or so. It is the responsibility of the BGO detectors to fill in the energy gap between the NaI detectors and the low energy threshold of the LAT. The BGO detectors are placed on opposite sides of the GLAST spacecraft so that at least one will be illuminated by any given event. Unlike the thin crystals making up the NaI detectors, the BGO detectors are 5 in diameter by 5 in high right circular cylinders, with nearly omni-directional response.

As a context instrument, GBM will observe GRBs (and other transients such as Solar flares as they occur) in the traditional 'hard X-ray band' between roughly 25 and $300 \mathrm{keV}$, where many of the global properties of bursts have 
been characterized quite well by previous missions, such as BATSE. Location, duration, peak flux and fluences have all been tabulated for bursts observed in this energy range. Any new catalog of bursts such as may be produced by GLAST should be compared with the current best sample of burst statistics (Briggs et al. 2003). This is quite difficult to do when the energy ranges do not overlap in the slightest, as would be the case for bursts observed by the LAT alone. The spectral characteristics that most bursts share involve the distributions of burst spectral model parameters (Preece et al. 2000). Most burst spectra are typically fitted to four-parameter empirical models (see the dicussion in section 2.1 below), with the possible exception of a second lowenergy component in some bursts (Preece et al. 1998a).

\section{GRB Science with GLAST}

Although the LAT will determine the locations of GRBs on the sky with very good accuracy, that will not be its primary scientific goal. As will be seen, GLAST's mission is primarily related to spectroscopy, as the energy coverage spans from $\sim 5 \mathrm{keV}$ to $300 \mathrm{GeV}$. If simultaneous observations with Swift are possible, the lower energy becomes $.2 \mathrm{keV}$; in any case, GLAST will represent an extraordinary leap forward for GRB spectroscopy. The burst community anticipates that by the launch of GLAST, Swift will have provided large numbers of accurate GRB localizations that will be used to determine distances from optical spectra of host galaxies. Indeed, the Swift mission should be a 'red-shift' factory, giving us good statistics for the distance distribution of GRBs for the first time. The scientific instruments on board Swift will not have the ability to observe bursts above $150 \mathrm{keV}$, thus a number of interesting scientific questions will remain open for the GLAST mission to resolve.

\subsection{Distribution of the Break Energy}

One of the first important spectral observations reported by BATSE was that typical GRB spectra require at most four parameters for successful spectral model fitting (Band et al. 1993). These are: the amplitude $A$ at fixed energy (usually $100 \mathrm{keV}$ ); two power law segments, indicated by the low- $(\alpha)$ and highenergy $(\beta)$ power law indices; and the energy of the break between the two power law segments, usually expressed as the peak in the $\nu \mathcal{F}_{\nu} \operatorname{spectrum}\left(E_{\text {peak }}\right)$. The two spectral indices $\alpha$ and $\beta$ are usually expressed in photon units, so a spectral index of -2 is flat in the $\nu \mathcal{F}_{\nu}$ representation. As a result, $E_{\text {peak }}$ can not be defined for $\beta>-2$, even though a spectral break exists between the $\alpha$ and $\beta$ power-law segments. It is this break energy, $E_{\mathrm{break}}$, that is usually quoted instead.

The $E_{\text {peak }}$ distribution for 5030 independent spectral fits from 156 GRBs observed by BATSE is shown as a solid-line histogram in Figure 1. For reference, the distribution consisting of only those spectra where $\beta<-2$ has been plotted as a dotted line; the two are not different in any significant way. The $E_{\text {peak }}-$ $E_{\text {break }}$ distribution quickly falls off to either side of the peak value $\sim 240 \mathrm{keV}$; this is the so-called "narrowness" problem. Quite a few discussions have arisen concerning to which extent the instrument response and bandpass contributes to the width; however, some things are quite well known: the uncertainties in the 


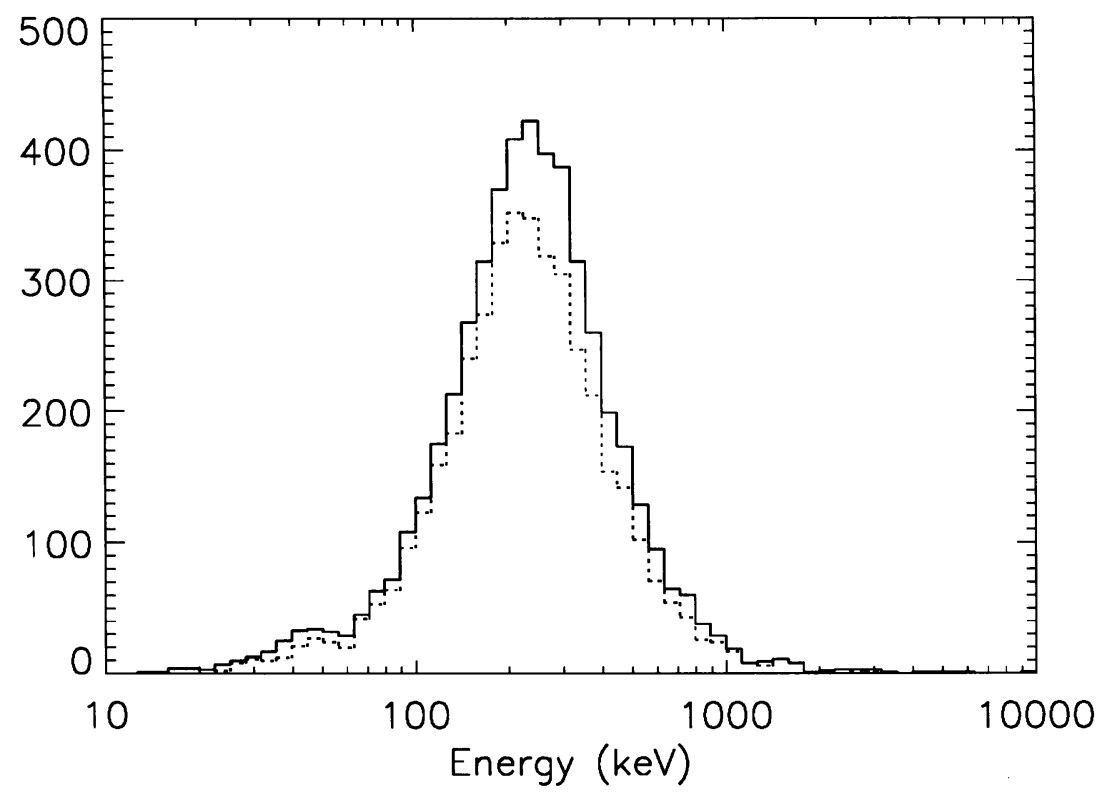

Figure 1. Distribution of $5030 E_{\text {break }}$ values from the BATSE Spectroscopy Catalog (solid). The dotted histogram shows the distribution of 4174 values of $E_{\text {peak }}$, where $\beta<-2$.

actual values are typically far smaller than the width of the distribution, so the peak, if it is an intrinsic property, is fully resolved in the data. Also, the peak of the instrument response curve is roughly at $100 \mathrm{keV}$, at which energy the $E_{\text {peak }}$ distribution has fallen by roughly one half, an indication that this portion of the distribution is real. Again, the high-energy wing of the distribution does not hide any large population of bursts with $E_{\text {peak }}>1 \mathrm{MeV}$, since such events were searched for, and not found, in the SSM data (Harris \& Share 1998) - given some very important caveats.

That the narrowness of the distribution should be a problem is immediately apparent from the fact that the break energy is transformed by a Doppler shift arising for any relative motion between the source and the observer. Indeed, different ranges in burst intensity yield different average values for the resulting distribution (Mallozzi et al. 1995), corresponding to the average cosmological redshift of the different brightness groups, assuming that weaker bursts come from farther away. Obviously, if bursts are not standard candles, the dispersion in red-shift within a single brightness group (the 156 bursts represented in Fig. 1 are the brightest) should account for at least a factor of $2-3$ in the width. What is worse, the spectra are thought to be emitted by shocked electrons in a relativistic blast-wave. Lorentz factors $\sim 100$ are required so that bursts avoid a pair-production runaway from photons above $2 \mathrm{MeV}$ (Hurley et al. 1994). Any 
dispersion in this blue-shift factor from burst to burst, as well as within each burst should also reflect

GLAST observations will be important for this problem in at least two ways. Nearly one fifth of all spectra in the BATSE Catalog have values that may lie in the LAT energy band: $\beta>-2$. Note that a -2 power law can not be extended higher in energy indefinitely, as this requires infinite energy at the source. The LAT will fully investigate the high-energy wing of the distribution, with counting statistics that will be good enough to determine the break energy unambiguously, especially with hard sources such as these. More generally, the LAT will assist in determining the high-energy power-law index very accurately, as it will lengthen the baseline of the observed power-law segment. With such good measurements of the high-energy power law, systematic errors in determining $E_{\text {peak }}$ within the GBM band will be reduced. Thus, the distribution of observed values will more accurately represent the intrinsic distribution, without the need for correcting for instrument response.

\subsection{Spectral Lag Measurements}

Norris et al. (2000) find a correlation between spectral lag and luminosity, where the spectral lag is defined by the peak in the cross-corellation between lightcurves in two broad energy channels $(25-50 \mathrm{keV}$ and $>100 \mathrm{keV})$ of counts observed by BATSE. Naively, one might expect the trend in lag to extend to higher energies. The LAT, in conjunction with the GBM should be able to verify this. If so, with a larger baseline, the lags should be easier to determine. If the lag-luminosity correlation holds up after repeated observations of bursts where the red-shift determination has been aided by good localizations from Swift, then one should be able to infer the distance to each source from the lag alone. Any improvement in the determination of the spectral lag will contribute to the accuracy of the red shift. Salmonson and Galama (2002) have made that case that the lag is related to kinematics of the bursting outflow: the Lorentz factor of the emitting material and the jet opening angle, which may be found from extended observations of the burst afterglow by Earth-based observatories.

\subsection{Pulse Narrowing and Lagging}

In addition to the spectral lag discussed above, individual pulses within a burst tend to get narrower with higher energy. Again, it requires the combination of BGM and LAT to determine if the trend typically continues into the LAT energy range. At some energy, one might expect to run out of photons, as the pulse has become too narrow to observe efficiently; in which case, there should be a discernable spectral break at an energy likely to lie within the LAT band pass. Detailed observations of the pulse narrowing will place important constraints upon the various models for spectral evolution within bursts (Ryde \& Svensson 2002; Crider et al. 1999). The important parameters in these studies are the temporal and spectral power-law decay indices.

\subsection{High Energy Cut-off}

A good fraction of burst spectra (one in five) have high-energy power-law indices greater than -2 , which, as noted above, clearly indicates a spectral break above $2 \mathrm{MeV}$. This is clearly seen in Figure 2 from the BATSE spectral observations. 


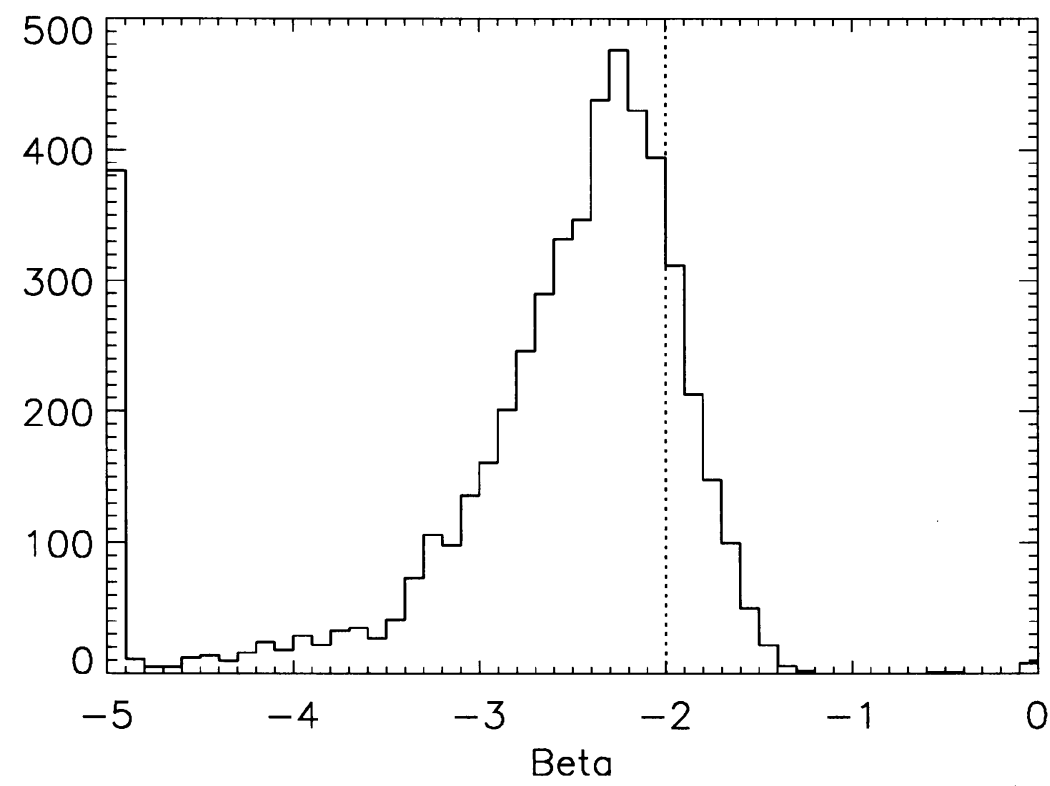

Figure 2. Distribution of 5038 values of $\beta$ from the BATSE Spectroscopy Catalog (solid). The lowest bin serves as an overflow for those spectra where $\beta<-5$, in addition to the 247 spectra that were consistent with $\beta=-\infty$ (exponential cut-off).

The current models of GRB emission predict a high-energy spectral break at an energy where losses by synchrotron emission just balances shock acceleration of the emitting electrons. Such a high-energy break, regardless of the high-energy power-law index, has not yet been observed. Detection of this break by the LAT will have direct consequences on GRB modeling, since the acceleration rate is unknown, but could be related directly to the known synchrotron loss rate at the observed energy. Because of its sensitivity, non-detection by the LAT has certain consequences as well, especially for low-intensity bursts. If an upper limit from the LAT falls below the extrapolation of a power-law fit of the high-energy GBM data, one can be certain that a spectral break lies somewhere in the transition region between the GBM and LAT, where the GBM is least sensitive.

\subsection{NHE Burst Spectra}

The lowest bin of the $\beta$ distribution presented in Figure 2 indicates that substantial numbers of burst spectra show very little evidence of a non-thermal power-law at high energies. These may correspond with the 'NHE' (no highenergy) spectra described by Pendleton et al. (1997) in the BATSE data. The four channel count data was deconvolved directly into photon fluxes received in four broad energy ranges, with the NHE spectra defined as having no flux above $\sim 300 \mathrm{keV}$. The distribution in $\beta$ clearly includes spectra that fall with 
increasing energy very quickly above the break energy; whether this translates into an absence of flux at $\mathrm{MeV}$ energies and above can only be determined by an instrument with the sensitivity of the LAT. Again, LAT upper limits on detection of photons within its energy range will prove to be useful constraining the high-energy spectral behavior. Some bursts have been classified as NHE, with no photons detected in their integrated flux data above $300 \mathrm{keV}$. The NHE bursts are important in that they seem to constitute a class of objects that are distributed in space more homogeneously than the total sample. For these, especially, it would be very interesting to know how well this trend extrapolates into the GBM BGO and LAT energy ranges. In the absence of photons from the primary spectrum, a Compton component (see below) would be relatively easier to discover, as the background from the burst itself would be very low.

\subsection{Compton Component}

Theoretical considerations of efficiency make synchrotron emission from relativistic shocked electrons the most plausible candidate for the source of GRB spectra (see review by Piran 1999). Although several aspects of the observed spectra have difficulties with the most simple formulation of this scenario (optically thin synchrotron; see Preece et al. 1998b \& 2002), taken at face value, the geometry is ripe for Compton upscattering of any ambient radiation (external inverse Compton - EIC) or of the primary burst spectrum itself (synchrotron self-Compton - SSC). The resulting broadband power density spectrum (PDS, or, $\nu \mathcal{F}_{\nu}$ ) should exhibit two well-separated peaks, with the separation related to the square of the bulk Lorentz factor of the shocked electrons. Similar behavior has been observed in the PDS of astrophysical jet sources (many references) as well as in the PDS of GRB afterglow spectra (Harrison et al. 2001).

There is no direct evidence for an IC component in the burst observations by EGRET, which was the only instrument that had the potential to make such an observation; however, this may be more due to the limitations of EGRET, most especially the large deadtime, than to any intrinsic feature of GRBs. There was an intriguing observation of a single event by EGRET that had a single 18 $\mathrm{GeV}$ photon 90 minutes after the burst trigger (Hurley et al. 1994). One could interpret such a photon as the tip of a "Compton iceberg"; the only recorded evidence of a spectrum that may have peaked at roughly $18 \mathrm{GeV}$ and at just that time, relative to the burst. The timing may have more to do with burst afterglow properties, than to the prompt emission, since it is easy to imagine an X-ray afterglow peaking 90 minutes after the gamma-ray trigger in the current external blastwave model. It should be mentioned that an obervation of $\mathrm{TeV}$ emission from GRB 970417a has been claimed by the Milagrito experiment (Atkins et al. 2000). Based upon the relative fluence between the observed $\mathrm{TeV}$ and the high-energy power-law behavior of the BATSE emission, if the same object is the source for the emission at both energies, a PDS with two separate spectral components is the most likely explanation (Atkins et al. 2003).

Where EGRET saw a single photon, the LAT will see hundreds, so that this mystery will be resolved. This will be especially true if concurrent observations can tie together any observed high-energy emission with the temporal behavior of the afterglow. The lack of any such corellation might signal a Comptonization of the prompt emission. Any such temporal lags, along with the relative weights 
between the two spectral components will yield a bonanza of information about the mechanisms at work in the source.

\section{Conclusion}

A wealth of observations of GRBs in the afterglow phase have been taken in the X-ray, optical and radio bands and thus much has been inferred of the possible physics of the emission source. A quite consistent model of blastwave shocked electrons, emitting synchrotron, has emerged from this intense broadband scrutiny. The GLAST mission is designed to bring broadband spectroscopy to bear upon the puzzle of the mechanism behind the prompt emission of bursts for the first time. Since the capabilities of the LAT will be so groundbreaking, we can not anticipate much of the new science that will come of these new observations.

Acknowledgments. The author would like to acknowledge the GLAST LAT web page

http://www-glast.stanford.edu/Instrument.html

from which much of the LAT description has been drawn.

\section{References}

Atkins, R. et al. 2000, ApJ, 533, L119

Atkins, R. et al. 2003, ApJ, 583, 824

Band, D. L. et al. 1993, ApJ, 413, 281

Briggs, M. S. et al. 2003, in preparation

Crider, A. et al. 1999, ApJ, 519, 206

Harris, M. J. \& Share, G. H. 1998, ApJ, 494, 724

Harrison, F. A. et al. 2001, ApJ, 559, 123

Hurley, K. et al. 1994, Nature, 372, 652

Mallozzi, R. S. et al. 1995, ApJ, 454, 59

Norris, J. et al. 2000, ApJ, 534, 248

Pendleton, G. N. et al. 1997, ApJ, 489, 175

Piran, T. 1999, Physics Reports, 314, 575

Preece, R. D. et al. 1998a, ApJ, 496, 849

Preece, R. D. et al. 1998b, ApJ, 506, L23

Preece, R. D. et al. 2000, ApJS, 126, 19

Preece, R. D. et al. 2002, ApJ, 581, 1248

Ryde, F. \& Svensson, R. 2002, ApJ, 566, 210

Salmonson, J. D. \& Galama, T. J. 2002, ApJ, 569, 682 